\title{
Experimental and Computational Study of Trapped Vortex Combustor Sector Rig with High-Speed Diffuser Flow
}

\author{
R. C. Hendricks, ${ }^{1}$ D. T. Shouse, ${ }^{2}$ W. M. Roquemore, ${ }^{2}$ D. L. Burrus, ${ }^{3}$ B. S. Duncan, ${ }^{3}$ \\ R. C. Ryder, ${ }^{4}$ A. Brankovic, ${ }^{4}$ N.-S. Liu, ${ }^{1}$ J. R. Gallagher, ${ }^{1}$ and J. A. Hendricks ${ }^{5}$ \\ ${ }^{I}$ NASA Glenn Research Center, Cleveland, Ohio, USA; ${ }^{2}$ Wright-Patterson Air Force Base, Dayton, Ohio, \\ USA; ${ }^{3}$ General Electric Aircraft Engines, Cincinnati, Ohio, USA $;{ }^{4}$ Flow Parametrics, LLC, Bear, \\ Delaware, USA; ${ }^{5}$ Diligent Design, Toledo, Ohio, USA
}

The Trapped Vortex Combustor (TVC) potentially offers numerous operational advantages over current production gas turbine engine combustors. These include lower weight, lower pollutant emissions, effective flame stabilization, high combustion efficiency, excellent high altitude relight capability, and operation in the lean burn or RQL modes of combustion. The present work describes the operational principles of the TVC, and extends diffuser velocities toward choked flow and provides system performance data. Performance data include EINOx results for various fuel-air ratios and combustor residence times, combustion efficiency as a function of combustor residence time, and combustor lean blow-out (LBO) performance. Computational fluid dynamics (CFD) simulations using liquid spray droplet evaporation and combustion modeling are performed and related to flow structures observed in photographs of the combustor. The CFD results are used to understand the aerodynamics and combustion features under different fueling conditions. Performance data acquired to date are favorable compared to conventional gas turbine combustors. Further testing over a wider range of fuel-air ratios, fuel flow splits, and pressure ratios is in progress to explore the TVC performance. In addition, alternate configurations for the upstream pressure feed, including bi-pass diffusion schemes, as well as varia-

Received in final form on 12 July 2001.

The authors would like to thank Dr. Marvin Goldstein, NASA Glenn Research Center Strategic Research Fund, the Air Force Research Laboratory (AFRL), and GE Aircraft Engines (GEAE) for their assistance in making this research possible.

Address correspondence to Robert C. Hendricks, MS 5-9, NASA Glenn Research Center, 21000 Brookpark Road, Cleveland, OH 44135. E-mail: Robert.C.Hendricks@grc.nasa.gov tions on the fuel injection patterns, are currently in test and evaluation phases.

Keywords Combustion, CFD, Trapped vortex combustor, Emissions, Combustor performance

\section{INTRODUCTION}

The trapped vortex combustor (TVC) is a novel design concept radically departing from the conventional swirl stabilized gas turbine engine annular combustor design approach that has been in use for the past 40 years. In the TVC concept, combustion is stabilized within cavities that are incorporated into the combustor liners. The cavities are designed to trap a vortex flow structure established through the use of driver air jets located along the cavity walls. Some amount of the overall combustor fuel is injected into the cavities where it mixes and burns quickly in the stable trapped vortex flow structure. The hot gases from these stable combustion zones exhaust from the cavities along radial struts spanning the gap between the inner liner and outer liner cavities. These hot gases then spread out across the face of the dome serving as pilots for igniting the remaining fuel that is injected into the main air stream entering the burner through the dome. The TVC design approach allows use of simple fuel injection techniques. It has demonstrated significantly enhanced ignition and combustion stability characteristics, provided high combustion efficiency over a much wider operating range, and provided significant reductions in pollutant emissions.

The TVC concept has been pioneered and developed by the Air Force Research Laboratory (AFRL) and GE Aircraft Engines (GEAE) under the joint sponsorship of AFRL, the Navy, and the Strategic Environmental Research Development Program (SERDP) (Roquemore et al., 2001). As part of this development effort, AFRL and GEAE jointly designed, fabricated, 
and tested a full scale 12-inch-wide rectangular prototypical TVC sector rig capable of operation at elevated inlet pressures and temperatures. A large quantity of performance data has been collected while running this prototypical test rig at operating conditions representative of typical conventional aircraft gas turbine engine applications (Burrus et al., 2001).

In this NASA-sponsored research program, the TVC 12-inch sector test rig was operated at high inlet velocities similar to those obtained in high flight speed applications. Unlike practical high-speed combustion systems that use ram air, high pressure drops across the combustor were used to increase the burner flow through velocity. Although this is not a practical approach for a practical vehicle, it does provide insights into the combustion process with high-speed inlet flows. The objectives of this work are 1) to extend the operational range of the TVC for high speed applications, 2) to establish a basis for numerical code evaluations by developing a database of performance characteristics and operational behavior of the TVC under conditions representing high through-flow velocities, and 3) to apply and assess the capabilities of an advanced multidimensional compressible reacting flow computational fluid dynamics (CFD) code to qualitatively and quantitatively capture the flow field and physics within the operating TVC. This paper presents some of the results from these early experimental investigations along with results from some of the initial CFD modeling calculations.

The experiments were simulated using a computational fluid dynamics (CFD) solver developed to compute multiphase, chemically reacting flows in complex geometries, such as those in aircraft gas turbine combustors. The FPVortex ${ }^{\mathrm{TM}}$ flow solver is applied to compute three cases of interest for the TVC test rig. Distinct fueling schemes were selected for the CFD runs, to illustrate differences in aerodynamic and combustion flow structures. Details of the CFD simulations are provided, and are discussed with regard to the photographic results obtained on the TVC rig at the same fueling conditions.

\section{BACKGROUND}

\section{Trapped Vortex Combustor}

The experimental apparatus used was an available 12-in wide rectangular (planar) sector rig that was originally designed, fabricated, and tested by a team consisting of representatives from the Air Force Research Laboratory (AFRL) and GE Aircraft Engines (GEAE). This same group of AFRL and GEAE representatives participated with NASA GRC representatives to conduct this current experimental testing. The TVC combustor rig is a planar sector rig consisting of 10 modules arranged linearly. Opposed upper and lower cavities are present, all with liquid spray pressure atomizing fuel injectors, numerous air jets, and film and slot cooling injections. The main combustor air is fed by a diffusion system consisting of three passages (tri-pass configuration), which split the incoming flow into approximately three equal flows. This flow enters the combustor at high, but subsonic speed. For the cavity-only fueled operating mode, only upstream plenum air flow passes through the diffuser passages. For the cavity-plus-main operating mode, main stream fuel is supplied to each passage via a pair of opposed liquid spray pressure atomizing fuel injectors. In the process of fueling the main stream, the fuel is injected into the high-speed cross-streams passing through the diffuser passages to provide some degree of premixing of the main fuel air before the mixture enters the combustion chamber for burning (Roquemore et al., 2001 and Burrus et al., 2001).

The 12-in rectangular TVC sector rig hardware is shown in Fig. 1. In this photograph one of the air cooled sidewalls has been removed for viewing. The arrow shown on the figure indicates flow from left to right. Station 3 (plenum) air enters into the diffuser, and is split into three streams by the diffuser struts at the upstream end of the tri-pass branches. The flow is diffused, however, as it must wrap around a fuel strut perpendicular to the flow. The fuel strut is airfoil-shaped, splitting the flow within each branch in half. Liquid spray simplex-type pressure atomizingfuel injectors are situated approximately midway down the fuel strut. When turned on, they inject directly into these tri-pass diffuser air streams. The upper and lower cavity fuel injection points (large openings on the cavity forward walls) can also be seen in the photo.

In the cavity only fueled-operating mode high-intensity combustion takes place and is largely confined to the cavity and the recirculation regions formed between the flows issuing from the tri-pass passages. For the cavity-plus-main operating mode, additional combustion takes place further downstream in the burner duct, as the liquid fuel from the main-stream injection evaporates and is ignited by the hot products transported out of the cavity.

Photographic results for the three cases under study here are shown in Figures 2, 3, and 4. Case 1 results, taken at an inlet pressure of $406 \mathrm{kPa}$, inlet temperature of $539 \mathrm{~K}$, overall equivalence ratio of $\phi=0.53$, and fueling split between the cavity $(\phi=0.65)$ and main feed system $(\phi=0.72)$, are shown in Fig. 2. Case 2 results, taken at an inlet pressure of $396 \mathrm{kPa}$, temperature of $506 \mathrm{~K}$, overall $\phi=0.52$, and fueling split between the cavity $(\Phi=1.86)$ and main $(\Phi=0.13)$, are shown in Fig. 3. Finally, cavity-only fueling was used for Case 3, with cavity $\phi=2.2$ and overall $\phi=0.52$, at an inlet pressure of $314 \mathrm{kPa}$ and temperature of $546 \mathrm{~K}$. These results are shown in Fig. 4. The rich fueling condition for the cavity results in highly intense combustion, with high fuel residence times, and overall high combustion efficiencies.

\section{Computational Fluid Dynamics Methodology}

The CFD modeling approach described in Ryder and McDivitt (2000) is followed in this work. An important aspect of this approach is to model the combustion system geometry precisely as it is installed on the test stand. The starting point in the process is development of a fully-detailed computer aided design (CAD) solid model representation of the hardware. The CAD solid model is preferably based on the same CAD model as that used for structural and thermal finite element analysis, 


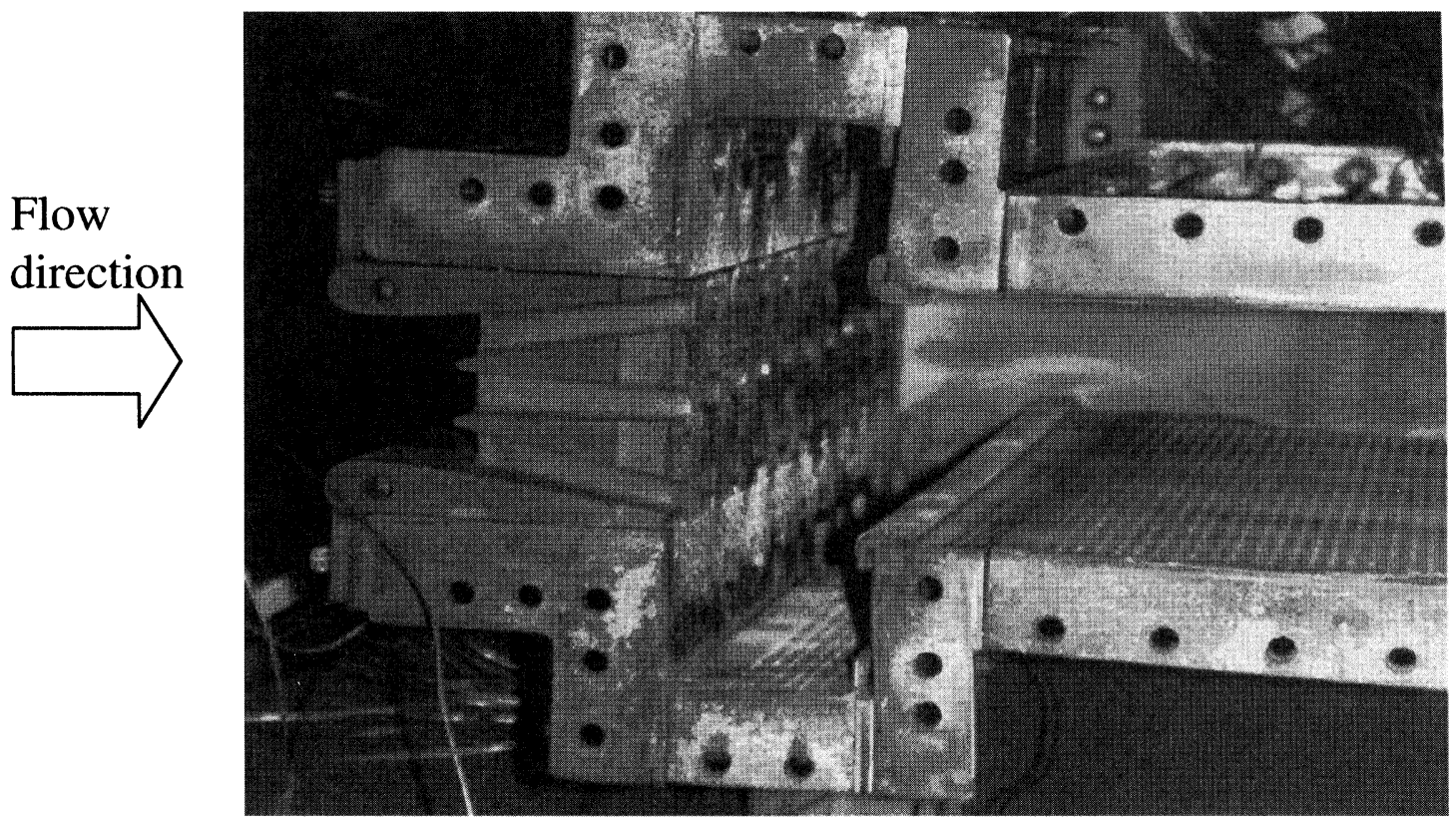

FIGURE 1

Test apparatus photo for the Trapped Vortex Combustor with tri-pass diffuser system, featuring a rectangular array of 10 fuel injector modules in a planar combustor sector rig. The near-field side wall has been removed for visualization. The fuel injectors can be seen on the rear plate of the cavity. Carbon deposits are visible on the ceramic combustor coating, reflecting extensive

hot-fired testing. Film cooling holes can be seen along the cavity walls, combustor core, and combustor liner walls.

and also for manufacturing. This approach has the benefit of including not only all the major flow hardware features, but also additional and miscellaneous hardware such as fasteners, bolts, support brackets, and other assembly components which

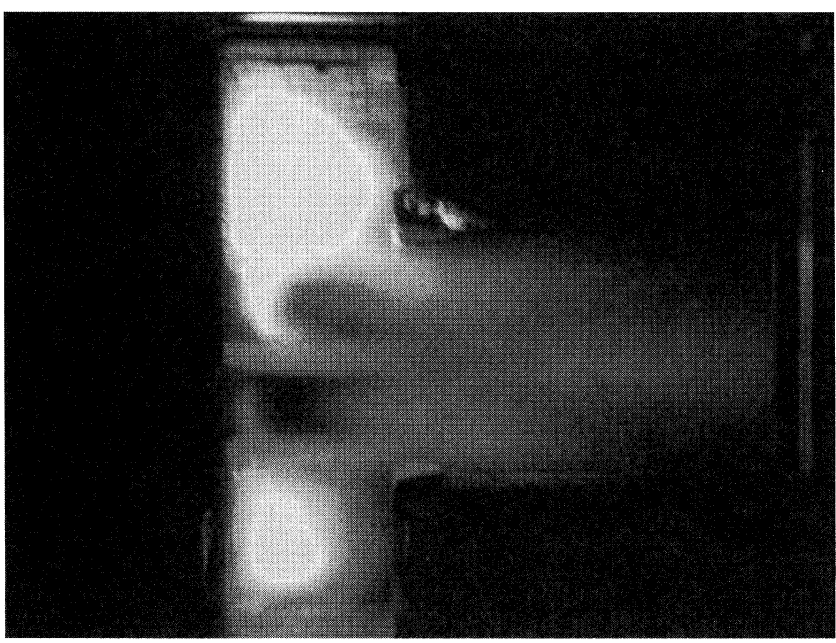

FIGURE 2

Photo of the Trapped Vortex Combustor with tri-pass diffuser, Run 2311. Inlet pressure $406 \mathrm{kPa}$ (58.8 psia); fueling into cavity and main feed arms. Cavity $\phi=0.65$, main feed arms $\phi=0.72$, overall $\phi=0.53 . \mathrm{T}_{3}=539 \mathrm{~K}\left(510^{\circ} \mathrm{F}\right)$; pressure drop $=56.4 \mathrm{kPa}(8.17 \mathrm{psi})$. can often adversely affect the flow distributions and quality. A further benefit of this approach is that all flow paths which are aerodynamically coupled will be identified and be included in the model. Once a satisfactory model has been created, an

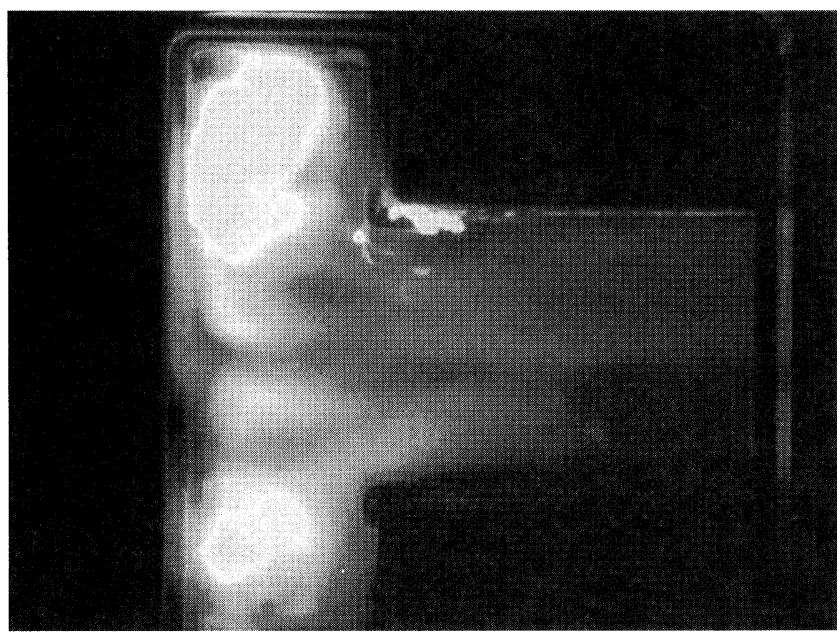

\section{FIGURE 3}

Photo of the Trapped Vortex Combustor with tri-pass diffuser, Run 2318. Inlet pressure $396 \mathrm{kPa}$ (57.4 psia); fueling into cavity and main feed arms. Cavity $\phi=1.86$, main feed arms $\phi=0.13$, overall $\phi=0.51 . \mathrm{T}_{3}=506 \mathrm{~K}\left(451^{\circ} \mathrm{F}\right)$; pressure drop $=53.3 \mathrm{kPa}(7.72 \mathrm{psi})$. 


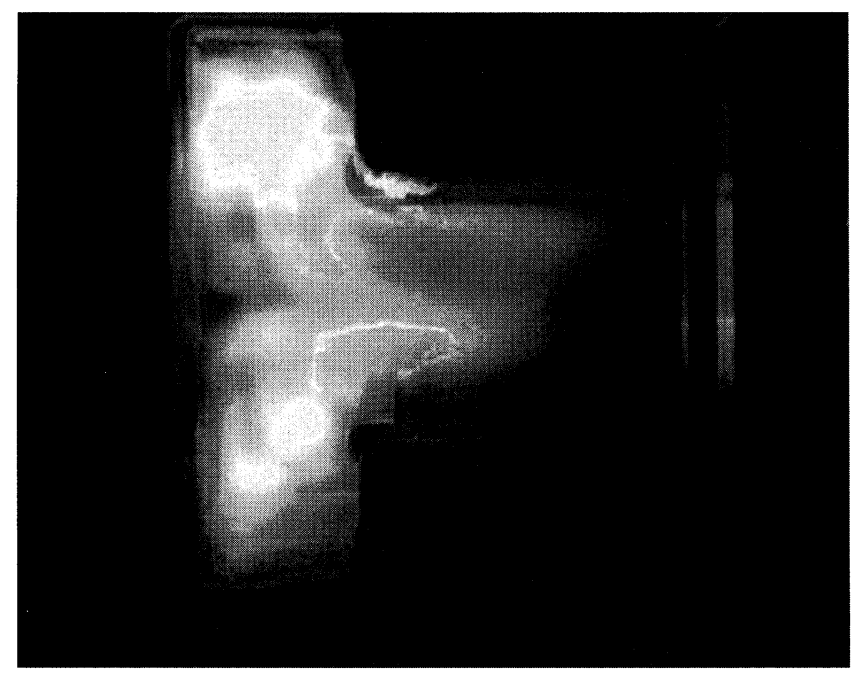

FIGURE 4

Photo of the Trapped Vortex Combustor with tri-pass diffuser, Run 2142. Inlet pressure $314 \mathrm{kPa}$ (45.8 psia); cavity fueling only. Cavity $\phi=2.2$, overall $\phi=0.53 . \mathrm{T}_{3}=546 \mathrm{~K}\left(522^{\circ} \mathrm{F}\right)$; pressure drop $=47.3 \mathrm{kPa}(6.86 \mathrm{psi})$.

"air-solid" representation of the hardware is created, using a Boolean operation. This is a solid model representation of the gas flow path. This model can then be automatically meshed using any of several commercial mesh generators, generally using tetrahedral elements. The resulting grid is tagged with boundary conditions, and outputted into an unstructured database read in by the flow solver. Intermediate results databases are created for assessing convergence, which can be viewed automatically in graphics post-processing packages. Further examples of this CFD methodology applied to gas turbine component flow fields may be found in the works by Ryder (2000) and Brankovic et al. (2001).

Physics-based modeling approaches are used to resolve the aerodynamics and combustion processes taking place within the combustion system. Turbulence is modeled using the twoequation or $\mathrm{k}-\varepsilon$ model of turbulence, together with wall functions. For combustion processes in which fuel-air mixing and flame stabilization are key phenomena to be resolved, turbulence wall functions provide adequate accuracy. If, however, pressure drop through the pre-diffuser or heat transfer along liner walls are of concern, near-wall grid clustering together with wall integration models of turbulence are used instead of wall functions.

The combustion process is modeled through detailed tracking of the liquid fuel spray droplets, evaporation, and chemical reaction. Individual spray droplets are tracked using a Lagrangian trajectory algorithm, developed and described by Raju (2000). The droplet model includes physics models for droplet drag, heat, and mass transfer to the liquid through established correlations. Droplet heating is modeled in detail, including the effect of internal circulation within the droplet, and temperature variations within the droplet. Droplet initial conditions are obtained from fuel injector experiments, and droplet initial velocity, radius, and temperature fields are specified for each injector. In the present case, the orifice injectors have been measured to produce $90^{\circ}$ conical spray droplet flow, and these results were used as the cavity and the main injector fueling initial conditions. Discrete liquid droplet streams are clocked at $10^{\circ}$ intervals, originating at the external lip of the fuel injector orifice. The droplet size distribution is modeled using a correlation taken from El Banhawy and Whitelaw (1980) applied to each droplet stream. The liquid spray droplet model is fully coupled to the aerodynamics and combustion models in the flow solver through continuity, momentum, and energy source terms. Typically, the smaller diameter droplets evaporate and react first, followed by the larger droplets, which penetrate further into the flow field.

A CFD simulation of the speed contour provides a convenient overview of the main aerodynamic features of the TVC with tri-pass diffuser system, and is shown in Fig. 5. The plenum conditions for the CFD simulations to be discussed differ from those in the photos in terms of the air mass flow rates through the diffuser and the secondary flow (or cooling air) paths. In the experimental runs shown in the photos, the tested mass flow rate resulted in peak flow velocities corresponding to Mach numbers near 0.7 within the diffuser branches. This velocity increase is expected to impact the computed temperatures within the combustor. For example, at the higher Mach number flow rate run in the experiment, shear velocity between the cross flow and the liquid fuel droplet would be greater, resulting in a higher fuel evaporation rate, and, consequently, more rapid combustion and heat release. CFD simulations with a monodispersed droplet distribution (60 micron diameter) gave lower droplet evaporation rates than would be anticipated. As such, some fuel would be still bound in the liquid droplets, and left the domain un-reacted when compared to a similar CFD solution with gaseous fuel. A Rosin-Rammler droplet distribution (bell-shaped) and particle tracking were applied to the simulation to mitigate these effects.

In Fig. 5, the aerodynamics definition sketch, the box drawn around the combustor indicates the region shown in the photographs in Figs. 2, 3, and 4. The upper figure cross section A-A offset from the combustor center plane clearly shows the interactions of the tri-pass diffuser airflows, with the cavity flow, with subsequent mixing in the downstream region. Air jets issuing into the upper and lower cavities are clearly seen, with rapid bending of the jets due to strong interactions with the combustion flow fields. Large-scale recirculation regions can be seen between the upper, middle, and lower branches of the tri-pass diffusion system. These recirculation regions hold hot products of combustion, which enter along the cavity back wall. These recirculation zones form exceptionally stable combustion regions, and are aerodynamically stable over a wide range of fuel-air ratios and inlet temperatures and pressures.

The lower figures show cross sections of the combustor taken at three axial positions within the cavity: near wall (1-1), midplane (2-2), and downstream wall (3-3), as indicated by the cross section cuts above. The high-speed jets are clearly observed, as 

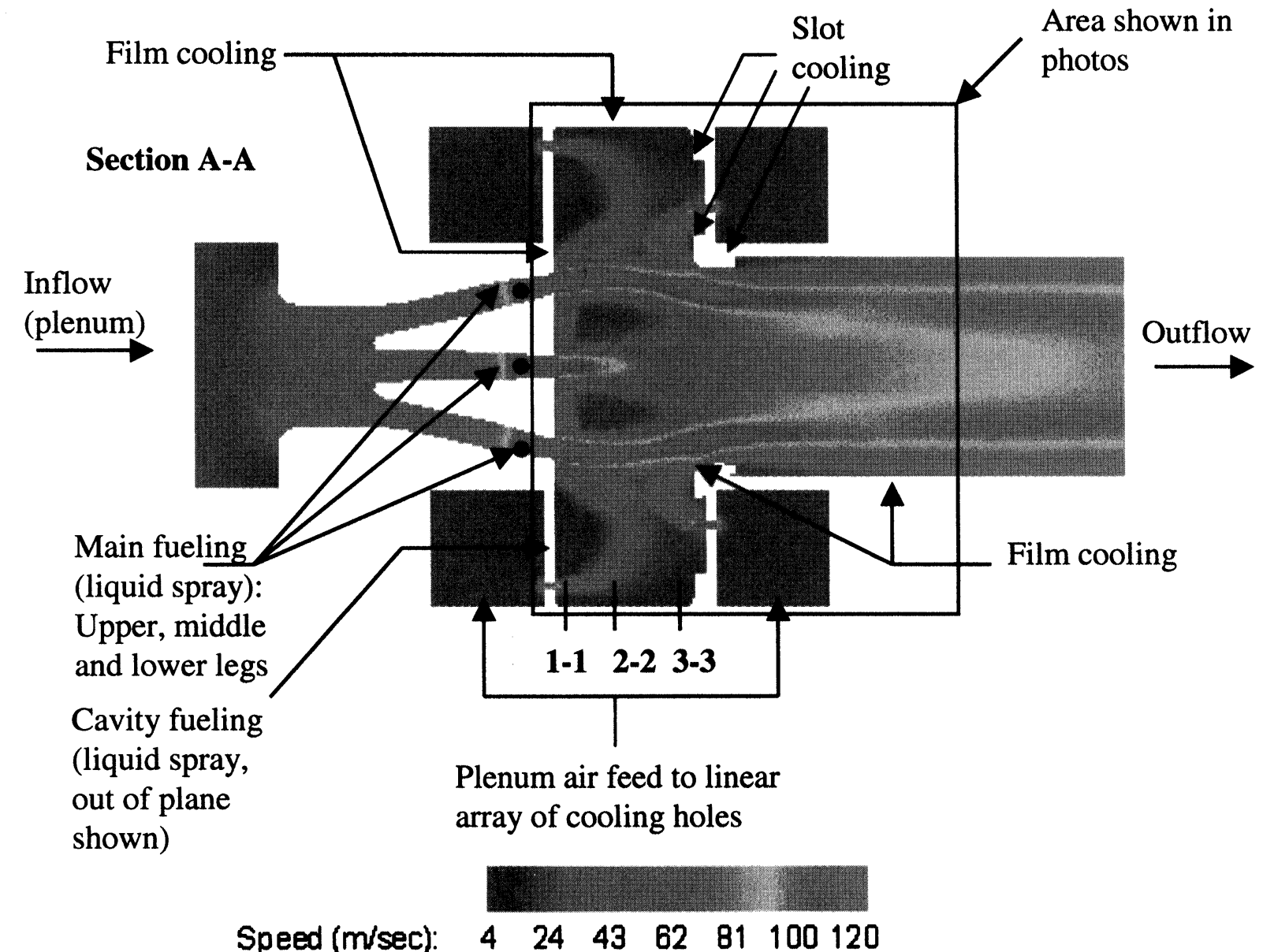

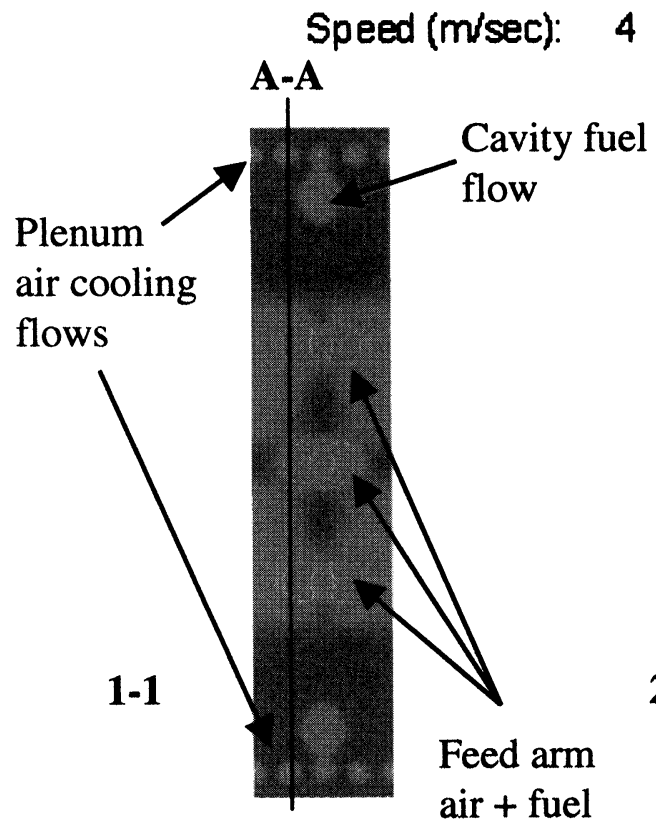

A-A flow

2-2

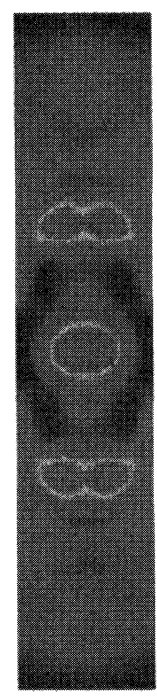

3-3

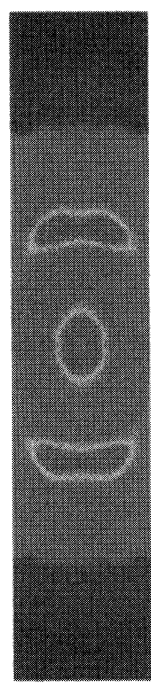

FIGURE 5

Speed contours and definition sketch for CFD simulation of the Trapped Vortex Combustor with tri-pass diffuser, showing air and fuel flows. Upper figure is a cross-section taken midway between combustor center-plane and end-wall. Lower figures represent cuts at three planes within the cavity. 
are the liquid fuel injection and the air jets issuing from the plenums. Although not easily observable in these views, significant amounts of slot and film cooling airflows are also entering into the cavity.

\section{RESULTS}

\section{Comparison of Combustor Simulations with Photographs}

\section{CASE 1 (Run 2311): Main and Cavity Fueling}

In Case 1, fuel was injected into both the main tri-pass branches and into the TVC cavities. The TVC cavity equivalence ratio was $\phi=0.65$, the main stream equivalence ratio was $\phi=0.73$, and the overall system equivalence ratio was $\phi=0.53$, based on a summation of all airflow in the cavity, the main stream, and downstream sections. The experimental photograph for the case is shown in Fig. 2, representing a timeand path-averaged spanwise view through all 10 sectors of the combustor. The small differences in $\phi$ are related to cavity and cooling air split estimations. The thermal-flow structure is represented in the photo through the luminosity of the flame. While not directly proportional to temperature, the observed flame luminosity shows high intensity burning within the cavity, particularly along the cavity back wall, as well as the central portion of the combustor. The flow simulation results for Case 1 are shown in Fig. 6, in terms of cross sections through the combustor center plane (Figs. 6(a) through (d), and axial cuts through the cavity (Figs. 6(e) through $(\mathrm{g})$ ).

The model calculation confirms that combustion is intense within the cavity, and particularly along the center plane, as seen in Figs. 6 (a) and (b). Hot products of combustion are entrained from the cavity region into recirculation regions between the tripass diffuser branches, and also along the downstream wall of the cavity, which wrap around a $90^{\circ}$ turn, mixing with the film cooling flow. In addition, with significant fueling into the main tripass diffuser passages, some combustion takes place in the near field of those flows. Most of the fuel entering through the main stream reacts further downstream in the straight duct, as seen by the hot streaks in Fig. 6. Strong mixing in the lateral or span wise direction takes place away from the center plane, with wellmixed high temperature flow throughout the straight duct. Interaction of the hot combustion gases with the liner film cooling and slot flow is clearly observed along the upper and lower portions of the combustor duct, flushed by a cool layer of fresh air.

The cross sections through the TVC cavity in Fig. 6(e) through (g) give further detail of the combustion flow structure. Near the cavity back wall, seen in Fig. 6(e), there is strong fluid coupling of the fuel jet with the upper, central air jet issuing from the plenum. This acts to enlarge the effective combustion area of the liquid jet stream. Figure 6(e) also shows the hot flow evolving from the outer tri-pass legs, indicated by the two hot spots. These are due to rapid combustion of the fuel as it enters the combustion chamber. Interestingly, the center passage of the tri-pass diffuser flow features no combustion at this plane, but instead, that flow experiences a significant combustion delay until that fuel-air mixture reaches the downstream duct. These overall features can also be observed in the luminosity photo of this case in Fig. 2. There is clearly a cooler zone in the central region, corresponding to the issuing of the center passage air-fuel stream, followed by a delayed combustion zone further downstream in the burner duct. Distinct combustion flow structures are seen in Fig. 2, including a hot streak due to flow from the upper tri-pass branch together with entrained flow from the cavity, and also the hot central jet which eventually reacts and combusts quickly once ignited. A distinct cool region also exists in the upper and lower right corners of the cavity, both in the experimental results and in the computational simulation. Also, intense burning along the back cavity wall is seen in the experiment and computational simulation.

\section{CASE 2 (Run 2318): Reduced Main Fueling, Increased Cavity Fueling}

In Case 2, fuel was again injected into both the trapped vortex cavity and through the tri-pass diffuser passages, but in different ratios. Inlet pressure and temperature were nearly the same as in Case 1 . The cavity was richly fueled, with local equivalence ratio of $\phi=1.8$, the main stream local equivalence ratio of $\phi=0.14$, and the overall system equivalence ratio of $\phi=0.52$. The experimental photograph for the case is shown in Fig. 3 , while the CFD simulation results for this case are shown in Fig. 7, in the same format as the cross sections in Fig. 6.

The combustion processes in both the cavity and in the central combustion zone are more varied than in Case 1. In the cavity, the rich fueling results in a strong interaction of the liquid fuel jet with the opposite cavity wall, with that fuel reacting quickly and intensely with incoming air flow from the cavity aft wall driver holes. The hot products of combustion associated with this interaction are purged rapidly into the downstream combustor section and interact with the slot and film cooling flow along the combustor duct. There is an indication that hot cavity flow comes in contact with the upper and lower cavity walls, despite significant film cooling and air jet flow. There is also a suggestion in the photo in Fig. 3 that this occurs in the rig as well.

The combustion process in the central combustor zone is also more varied than in Case 1. Larger combustion recirculation regions are present between the tri-pass diffuser outer and center passage flows, with hot flow also engendered from the tri-pass passages due to direct fueling. The shear layer formed between the cavity and tri-pass diffuser outer branch supports a high temperature gradient, separating the driven cavity flow and the high speed, slightly heated tri-pass jet flow. Rapid mixing of the combustion gases from the various sources, and with the film and slot cooling flows, occurs in the combustor duct further downstream.

\section{CASE 3 (Run 2142): Cavity Fueling Only}

In Case 3, fuel was injected into the trapped vortex cavity only, with no fuel injected into the diffuser passages. For this case, the cavity was richly fueled at a local equivalence ratio of $\phi=2.2$, while overall system equivalence ratio was maintained 


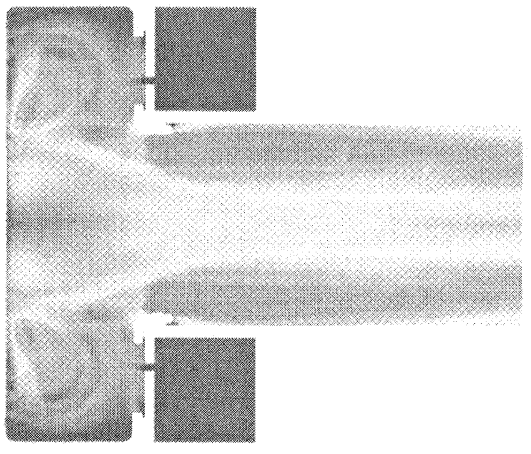

(a) Spanwise-averaged static temperature.

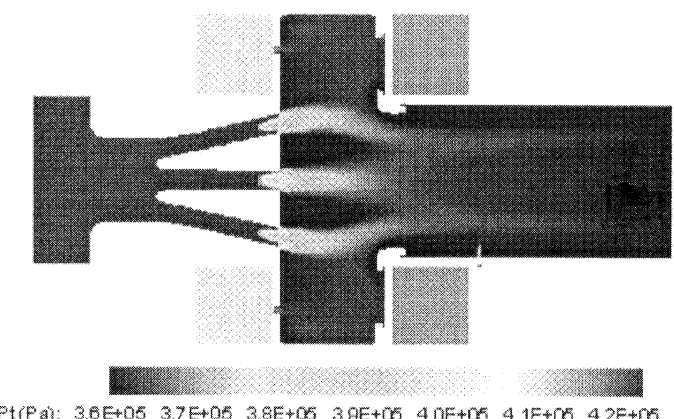

(c) Total pressure contour.

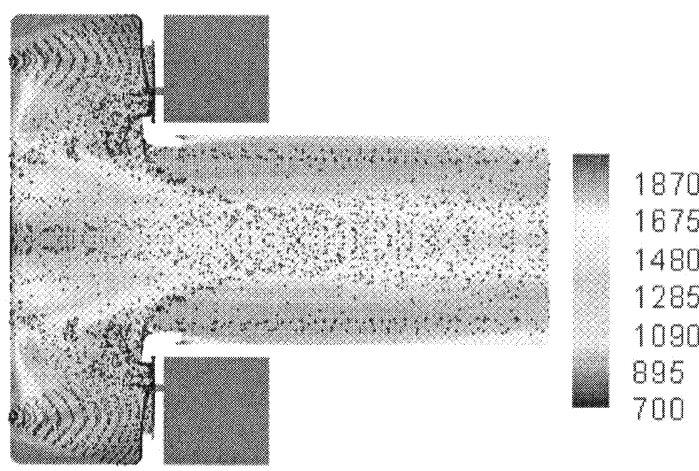

(b) Spanwise temperature with spray droplets.

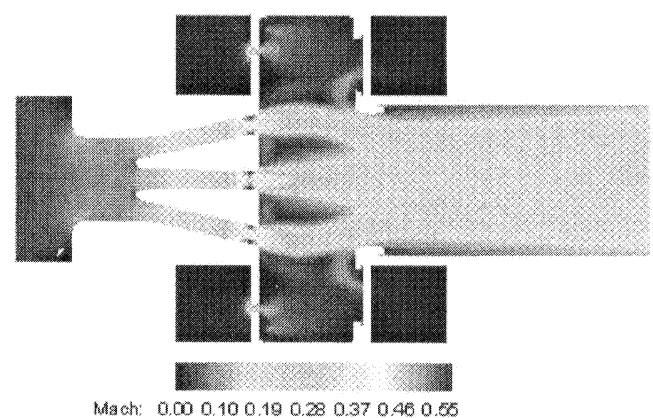

(d) Mach number contour.

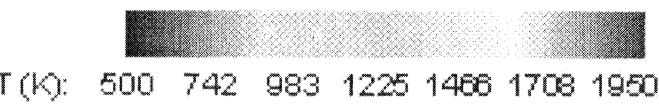

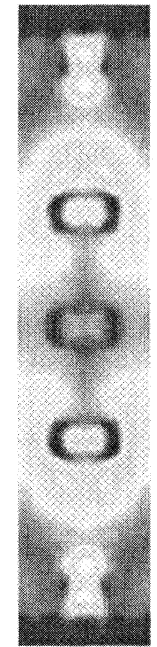

(e) $\mathrm{X}=0.001 \mathrm{~m}$ off upstream cavity wall

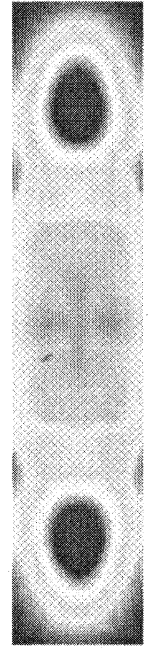

(f) Midway through cavity

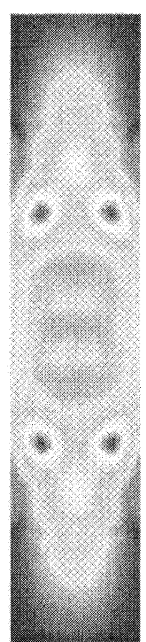

(g) $\mathrm{X}=0.001 \mathrm{~m}$ off downstream cavity wall

FIGURE 6

Computed temperature contours for Case 1. (a)-(d) Lengthwise cuts through the combustor. (e)-(g) Axial cuts through cavity. 


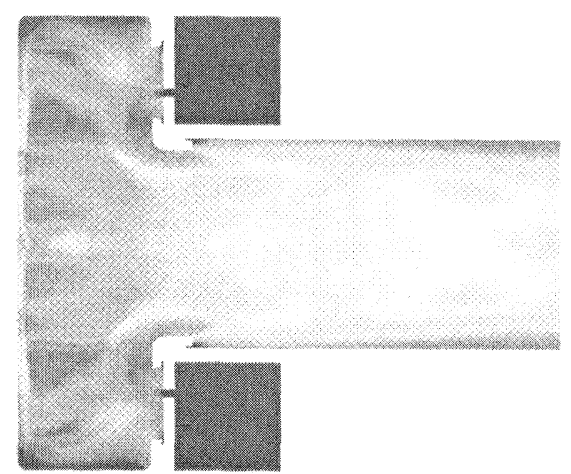

(a) Spanwise-averaged static temperature.

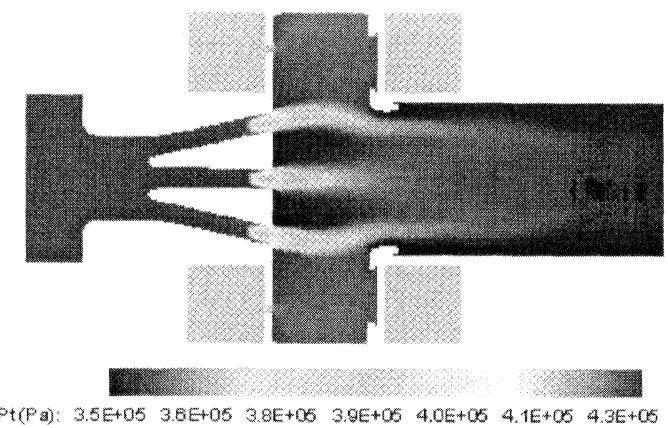

(c) Total pressure contour.

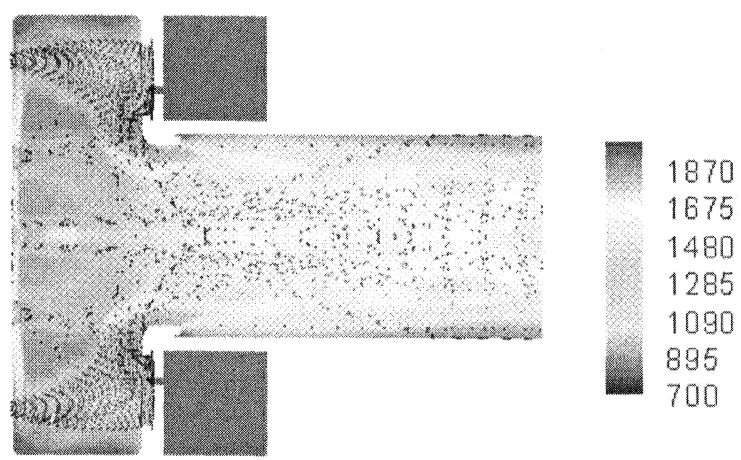

(b) Spanwise temperature with spray droplets.

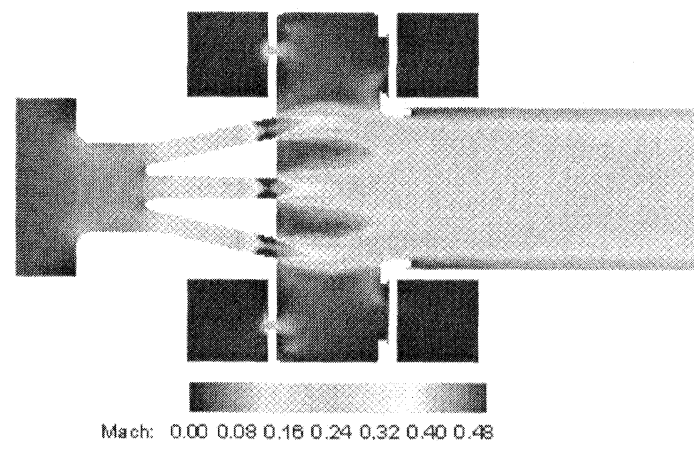

(d) Mach number contour.

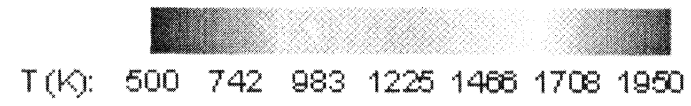

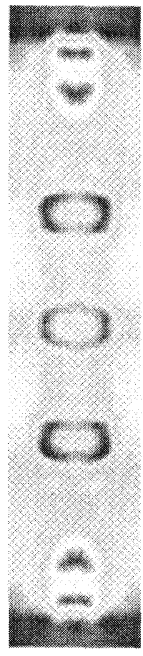

(e) $X=0.001 \mathrm{~m}$ off upstream cavity wall

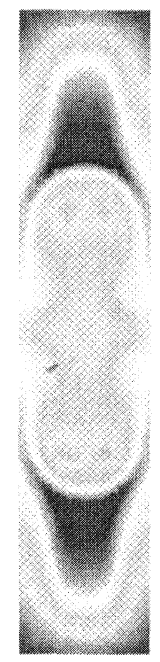

(f) Midway through cavity

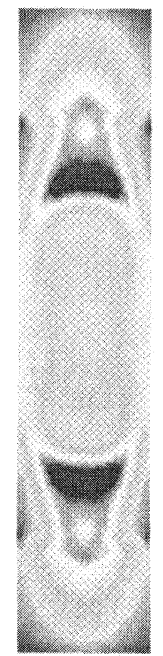

(g) $\mathrm{X}=0.001 \mathrm{~m}$ off downstream cavity wall

\section{FIGURE 7}

Computed temperature contours for Case 2. (a)-(d) Lengthwise cuts through the combustor. (e)-(g) Axial cuts through cavity. 


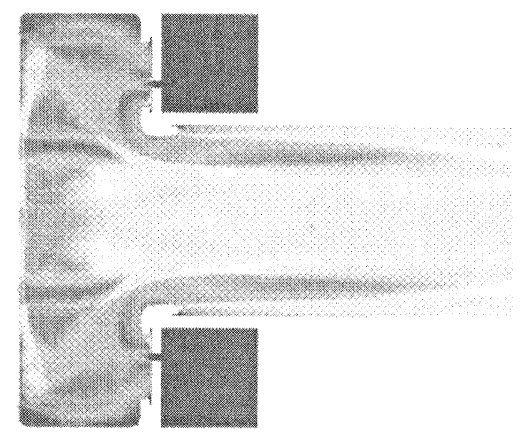

(a) Spanwise-averaged static temperature.

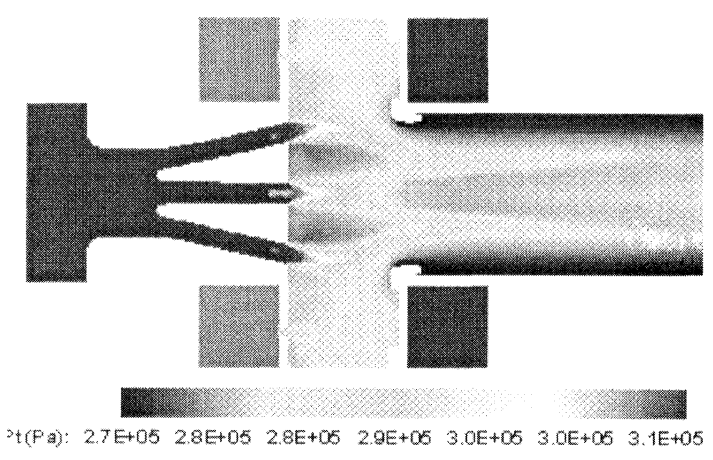

(c) Total pressure contour.

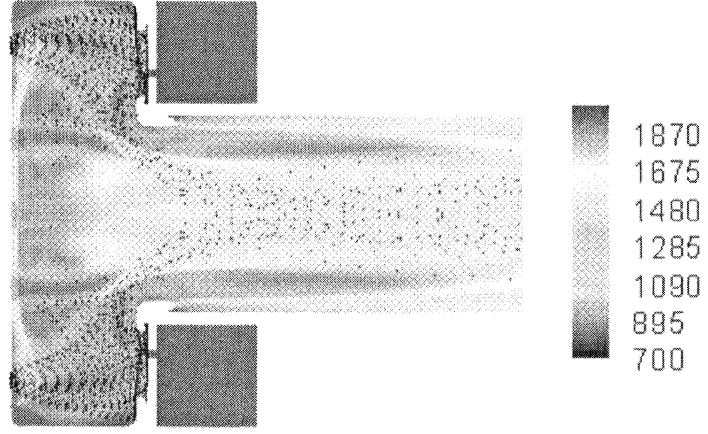

(b) Spanwise temperature with spray droplets.

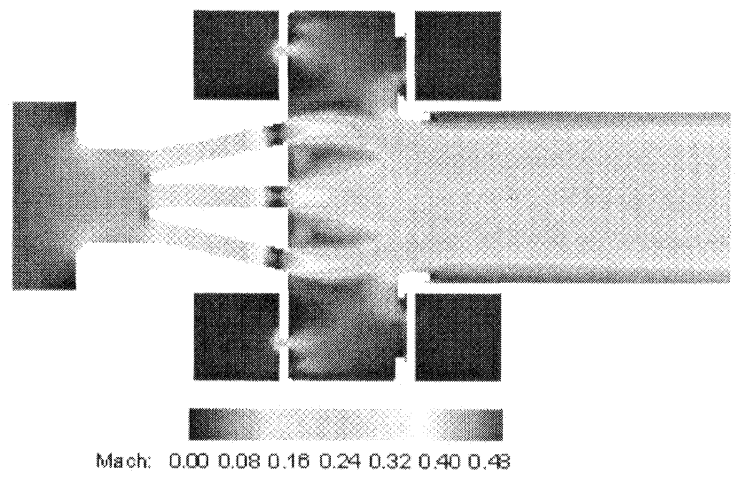

(d) Mach number contour.

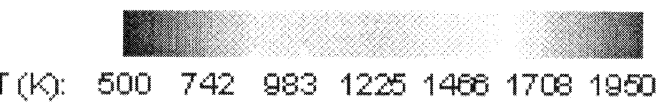

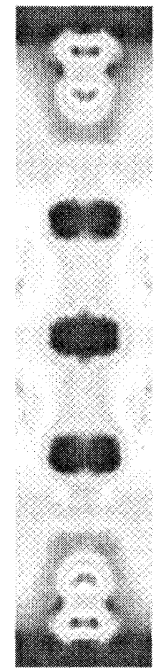

(e) $\mathrm{X}=0.001 \mathrm{~m}$ off upstream cavity wall

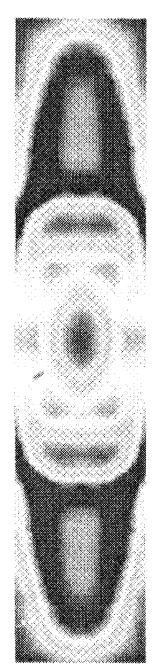

(f) Midway through cavity

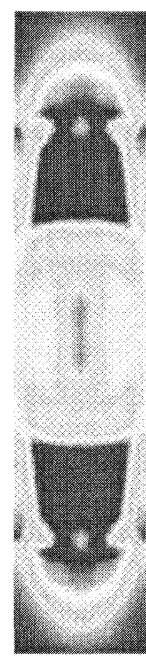

(g) $\mathrm{X}=0.001 \mathrm{~m}$ off downstream cavity wall

FIGURE 8

Computed temperature contours for Case 3. (a)-(d) Lengthwise cuts through the combustor. (e)-(g) Axial cuts through cavity. 
at $\phi=0.53$. Inlet pressure and temperature were again nearly the same as in Case 1. The experimental photograph for Case 3 is shown in Fig. 4, while the CFD simulation results for the case are shown in Fig. 8, in the same format as for the previous cases.

The combustion process for this case forms a highly intense combustion zone that originates entirely within the cavity. As in the other cavity flow fields, the flame is attached to the edges formed by the spray cone and anchored along the periphery of the hole near the injector orifice. The flame is turned by the various jet and film cooling flows and wraps around the tri-pass airflows, filling the recirculation regions between the outer, center, and inner passages of the tri-pass diffuser. These regions are also very hot, with products of combustion entrained from the cavities and the incoming tri-pass diffuser air flows. As in the previous cases, hot products mix out well in the downstream burner duct.

\section{Combustor Experimental Performance Data}

To establish the engineering performance parameters required for evaluation of the combustor, data were obtained for $\mathrm{NOx}$ emissions, combustion efficiency, and lean blow-out at different inlet temperatures, different fuel-air ratios, and different cavityto-main-stream fuel splits. The majority of the measured data were obtained in the cavity-only fueled operating mode. The measured NOx emissions have been corrected to standard day humidity levels.

Results for humidity-corrected EINOx versus burner bulk cold flow residence time are shown in Fig. 9. These results indicate that a factor of three reduction in the cold flow bulk residence time can reduce NOx emissions by $33 \%$ to $50 \%$. The observed

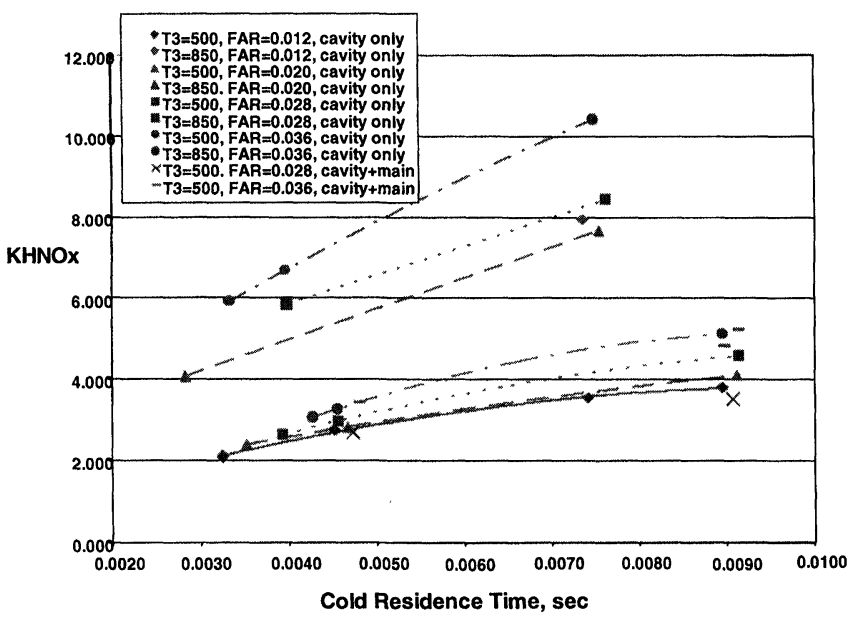

FIGURE 9

Experimental TVC-tri-pass data of humidity-corrected EINOx vs. burner bulk cold residence time, at various test conditions. Inspection of the plot reveals that NOx can be reduced from $33 \%$ to $50 \%$ of baseline values, and that residence times can be controlled over a range of 2 to 3 times. NOx is seen to be relatively insensitive to $\mathrm{T}_{4}$. Pressure drop performance at $\mathrm{P}_{4}$ is up to $20 \%$ to $25 \%$ of $\mathrm{P}_{3}$. Stable combustor operation observed over all parameter settings.

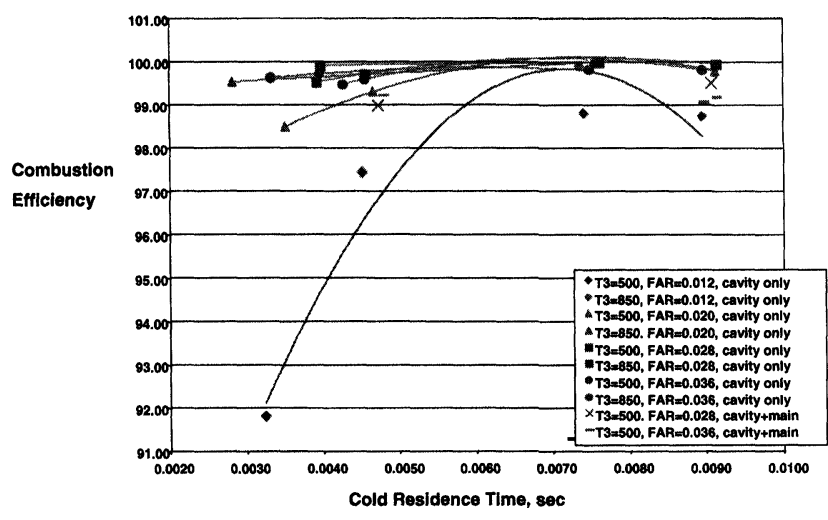

FIGURE 10

Experimental TVC-tri-pass data of combustion efficiency vs. bulk cold residence time. Combustion efficiency decreases with residence time, significantly at low fuel-air ratios. For example, less than $98.5 \%$ for $\mathrm{f} / \mathrm{a}<0.02$. Further measurements planned for the performance region of $0.012<\mathrm{f} / \mathrm{a}<0.02$.

$50 \%$ reduction in NOx emissions is in line with anticipated benefits of using TVC technology in advanced military gas turbine engine applications.

Combustion efficiency versus bulk cold flow residence time is shown in Fig. 10. High values of combustion efficiencies (>99\%) are achieved over almost the entire range of residence times, over different fuel-air ratios, and cavity-to-main fuel splits. The effect on measured combustion efficiency is significantly amplified for overall lean operation $(\mathrm{f} / \mathrm{a}=0.012)$ at low operating inlet temperature $\left(500^{\circ} \mathrm{F}\right.$.).

Overall burner exit equivalence ratio at lean blow-out (LBO) performance versus burner cold flow reference velocity is shown in Fig. 11. Values of 0.1 are generally considered good performance for conventional swirl-stabilized combustion devices, which typically operate at reference velocities of $50 \mathrm{ft} / \mathrm{sec}$ or less. The TVC generally performs at or below this value at reference velocities up to a factor of three higher, with most data points between the 0.06 and 0.08 levels. Based on prior test experience with this prototypical TVC test rig, it was anticipated that LBO performance would be exceptionally good for this device.

\section{SUMMARY AND CONCLUSIONS}

This paper described experimental testing and numerical simulation of a trapped vortex combustor rig with diffuser flows to local Mach numbers near 0.7 , with a view towards understanding the fundamental aerodynamic and combustion processes under different fueling conditions and evaluation of engineering performance factors, such as EINOx combustion efficiency and lean blow-out. Photographs of the combustor hardware and photographs of the observed flame luminosity under three differing cavity-to-main-stream fueling splits led to insights into flame structure and potential hot spots within the combustion zone. CFD simulation results for the liquid fuel spray droplet evaporation and combustion process showed in detail the complex 


\section{$\Phi$ Exit At Burner LBO}

( $\mathrm{T} 3=330 \mathrm{~F})$

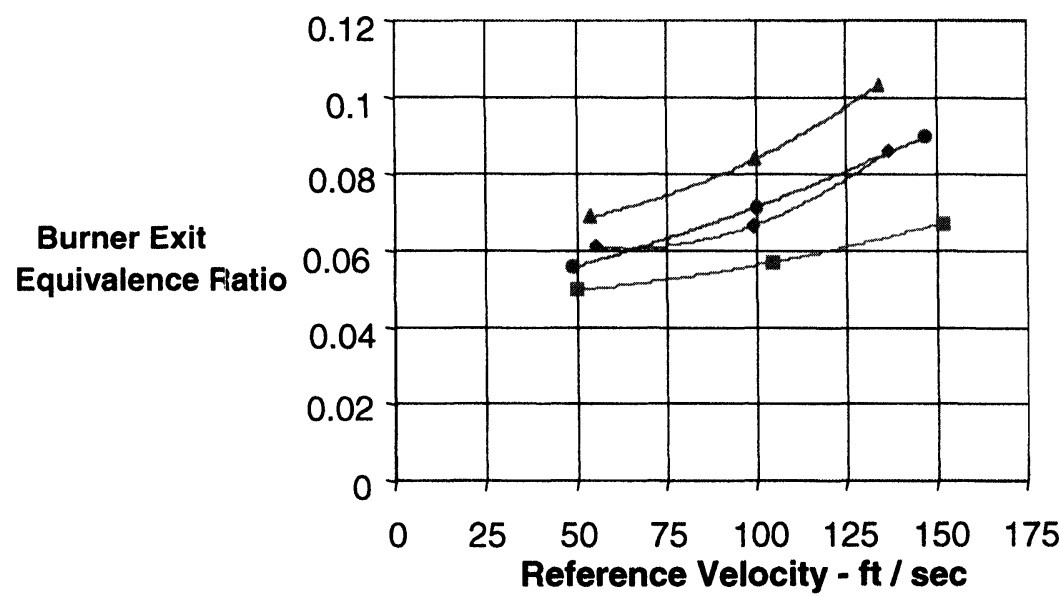

- Outer cavity @ 25 psia

- Outer cavity @ 50 psia

- Inner cavity @ 25 psia

- Inner cavity @ 50 psia

FIGURE 11

Experimental TVC-tri-pass data of combustor lean blow-out (LBO) vs. reference velocity, at $172.5 \mathrm{kPA}$ (25 psia) and $345 \mathrm{kPA}$ (50 psia). For this case, cavity $\phi=0.28$, overall $\phi=0.359$. A value of 0.1 is considered good performance for conventional gas turbine combustor LBO performance. Trend observed is that LBO increases as reference velocity increases.

aerodynamic flow interactions and captured the very different combustion processes at work in the combustor main and cavity zones. It was noted that the CFD runs with monodispersed droplet distributions (60 micron diameter) gave reduced droplet evaporation rates and, consequently, reduced overall combustion efficiency; the Rosin-Rammler droplet distribution (bellshaped) was applied to mitigate these effects. Additional experimental testing and data reduction showed the baseline TVC configuration with tri-pass diffuser to be very promising with respect to key performance parameters, and in comparison to values for conventional, swirl-stabilized combustors. The TVC combustor is continuing to undergo testing at AFRL and is being systematically and vigorously tested over a wider range of fueling and inlet conditions. Additional design work is also underway in tailoring the cavity and main stream fuel injection features in ways that will enhance fuel-air mixing and further improve performance characteristics of the TVC class of combustors.

\section{REFERENCES}

Brankovic, A., Ryder, Jr., R. C., Sturgess, G. J., Lee, J., Kushari, A., Lubarsky, E., Zinn, B. T., 2001, Computational and Experimen- tal Study of Aerodynamics and Heat Release in a Liquid Fueled Combustor, AIAA Paper 2001-0976.

Burrus, D. L., Johnson, A. W., Roquemore, W. M., and Shouse, D. T., 2001, Performance Assessment of a Prototype Trapped Vortex Combustor Concept for Gas Turbine Application, ASME IGTI Paper 2001-GT-0087, ASME IGTI Turbo Expo 2001, 4-7 June 2001, New Orleans, Louisiana.

El Banhawy, Y., and Whitelaw, J. H., 1980, Calculation of the Flow Properties of a Confined Kerosene-Spray Flame, AIAA J., vol. 18, no. 12 , pp. 1503-1510.

Raju, M. S., 2000, An Overview of the NCC Spray/Monte Carlo PDF Computations, AIAA Paper 2000-0337.

Roquemore, W. M., Shouse, D., Burrus, D., Johnson, A., Cooper, C., Duncan, B., Hsu, K.-Y., Katta, V. R., Sturgess, G. J., and Vihinen, I., 2001, Trapped Vortex Combustor Concepts for Gas Turbine Engines. AIAA Paper 2001-0483. 39th AIAA Aerospace Sciences Meeting and Exhibit, 8-11 January 2001, Reno, Nevada.

Ryder, Jr., R. C., 2000, Application of the National Combustion Code Towards Unsteady Mixing and Combustion Modeling, AIAA Paper 2000-0335.

Ryder, Jr., R. C., and McDivitt, T., 2000, Application of the National Combustion Code Towards Industrial Gas Fired Heaters, AIAA Paper 2000-0456. 

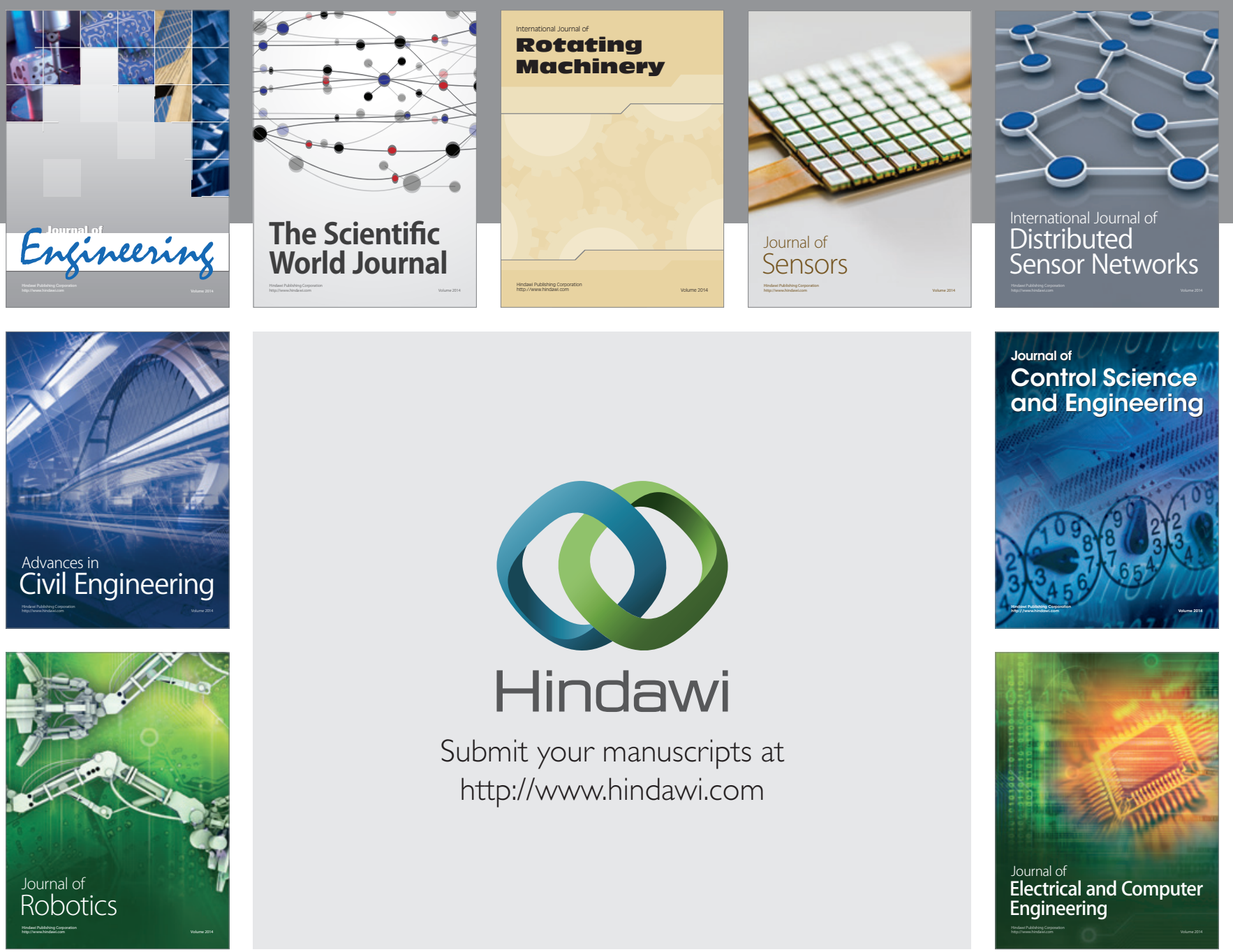

Submit your manuscripts at

http://www.hindawi.com
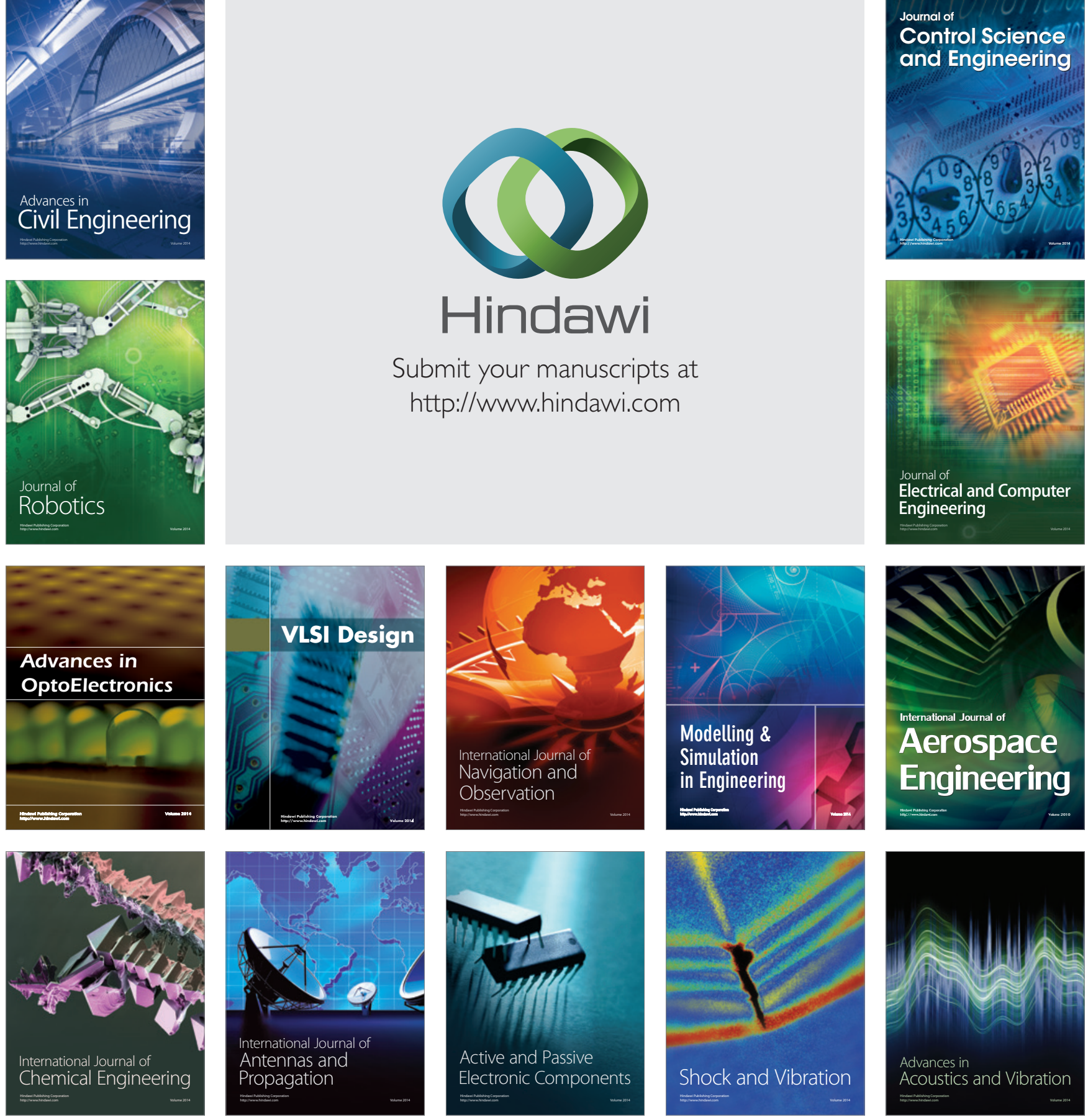